\title{
Numerical study of the structure flow of the gas-vapor mixture in a channel with injection of water droplets
}

\author{
Eugene Maslov $^{1, a}$, Irina Zharova ${ }^{2}$, Boris Borisov $^{1}$, and Natalya Fedotova ${ }^{1}$ \\ ${ }^{1}$ Tomsk Polytechnic University, Tomsk, Russia \\ ${ }^{2}$ Tomsk State University, Scientific Research Institute of Applied Mathematics and Mechanics, Tomsk, \\ Russia
}

\begin{abstract}
Mathematical modeling algorithm for the numerical analysis of the dynamics and evaporation of the water droplet which is injected into a gas-vapor flow is offered. The algorithm was tested with the help of the problem solution about a viscous fluid flow in a plane channel of constant cross section. It was shown that the proposed mathematical model and implementation algorithm are adequate to the physics of the investigated process. It was shown that the proposed mathematical model and implementation algorithm are adequate to the physics of the investigated process. Results of numerical study of the flow structure of the gas-vapor mixture in the channel with injection of water droplet under varying the initial droplet parameters are given.
\end{abstract}

\section{Introduction}

Technological processes in a number of systems of energy installations are related to the stream of the working medium as a multiphase flow. We need minimize thermal and hydraulic losses, and to provide a rapid and complete combustion of the fuel components, and to provide specified operating parameters of the outlet working medium for improve the efficiency of such systems. A detailed study of the occurring in heterogeneous multiphase flows processes allows to find the best of a constructive solution for the ultimate goal. Problem to define the basic flow parameters of the combustion products of organic fuels, in which water is present in a different states of aggregation is one of the most actual at the moment. Presence of water in the mixtures may be due to the thermochemical reactions to form of water vapor. Injection of fine droplets is carried out in a number of energy technologies to achieve the required thermodynamic flow parameters. In particular, the injection of water allows you to adjust gas-vapor mixture parameters at the inlet into the appropriate turbine area. The following problems arise in this case:

- it is necessary to exclude the possibility of droplet deposition on the channel walls;

- it is necessary to implement the conditions of the complete water evaporation;

\footnotetext{
${ }^{a}$ Corresponding author: maslov_eugene@mail.ru
}

This is an Open Access article distributed under the terms of the Creative Commons Attribution License 4.0, which permits unrestricted use, distribution, and reproduction in any medium, provided the original work is properly cited. 
Table 1. Characteristics of a medium.

\begin{tabular}{|l|l|l|l|l|}
\hline \multirow{2}{*}{ Fluid } & \multicolumn{3}{|c|}{ Characteristics } \\
\cline { 2 - 5 } & Density, $\mathrm{kg} / \mathrm{m}^{3}$ & $\begin{array}{l}\text { Dynamic } \\
\text { viscosity, } \mathrm{Pa} \cdot \mathrm{sec}\end{array}$ & $\begin{array}{l}\text { Thermal conductivity, } \\
\mathrm{W} /(\mathrm{m} \cdot \mathrm{K})\end{array}$ & Heat capacity, J/(kg $\cdot \mathrm{K})$ \\
\hline Glycerin & $1.26 \cdot 10^{3}$ & 1.49 & 0.28 & $2.43 \cdot 10^{3}$ \\
\hline Water & $10^{3}$ & $10^{-3}$ & 1 & $4.2 \cdot 10^{3}$ \\
\hline Air & 1.2047 & $18.2 \cdot 10^{-6}$ & 0.0257 & $1.005 \cdot 10^{3}$ \\
\hline
\end{tabular}

Table 2. The flow velocity in the initial section of the channel.

\begin{tabular}{|c|c|c|c|}
\hline & Glycerin & Water & Air \\
\hline$u_{\text {in }}, \mathrm{m} / \mathrm{sec}$ & 3.55 & $3 \cdot 10^{-3}$ & $4.53 \cdot 10^{-6}$ \\
\hline
\end{tabular}

- it is necessary achieve to homogenization working medium with a value of the dryness degree, which provide minimal mechanical flow impact on the turbine blades and the maximum value of the internal efficiency of the turbine.

Numerical modeling allows us to study the basic laws of processes in heterogeneous multiphase flows in power installations and to identify the mutual influence of various factors a priori. This allows to find new approaches to the implementation of promising engineering solutions during the design of energy systems.

The purpose of this work is the numerical analysis of the dynamics and of heat transfer when introducing water droplet with different initial conditions into a stationary saturated vapor flow or into superheated vapor flow.

\section{Mathematical modeling of the gas-vapor mixture flow in a cylindrical channel with injection of water droplets}

We consider the cylindrical channel of constant cross section. Numerical analysis was done on the basis of a mathematical model [1]. The fluid flow is described by the stationary Navier-Stokes equations for a compressible viscous heat-conducting gas. Droplet dynamics is described by the equation of motion in the velocity field of the steady flow of gas, taking into account the viscous drag forces and taking into account the gravity force. Thermal state of a drop is described by the thermal conductivity equation. The velocity of evaporation of a liquid from the surface droplet was determined by Knudsen-Langmuir relation. A flow was considered irrotational with parabolic velocity profile $u_{\text {in }}$ and constant temperature $T_{\text {in }}$ at the channel input. The adhesion and adiabatic conditions were specified at the channel output.

Numerical solution was implemented by control volumes method. Numerical solution was implemented by control volumes method. Boundary conditions on the channel surface were implemented with help of border functions by using empirical relationships for flow with low Reynolds numbers [2]. Differential equations were approximated by implicit Five-Points scheme. Scheme QUICK against the flow was used to approximate of the convective terms, procedure PISO was used to calculate the flow field. The diffusion terms were approximated by the central second order scheme.

The algorithm was tested with the help of the problem solution about a viscous fluid flow in a plane channel of constant cross section [3]. The results of mathematical modeling of flow near the wall of the channel were compared with the numerical results [3, 4] and experimental data [5]. The flows of fluids with different properties in a channel (see Table 1) were analyzed at a fixed value of the Reynolds number $\operatorname{Re}_{d}=\rho_{f} u_{i n} d / \mu_{f} \equiv 150$ (in analogy to [3]). Values of the characteristic size channel, $d=(0.03 \div 0.3) \mathrm{m}$, and of the flow velocity $u_{\text {in }}=\left(4.53 \cdot 10^{-6} \div 3.55\right) \mathrm{m} / \mathrm{sec}$ (Table 2$)$ were varied in accordance with a predetermined value Re. 
Thermophysical Basis of Energy Technologies

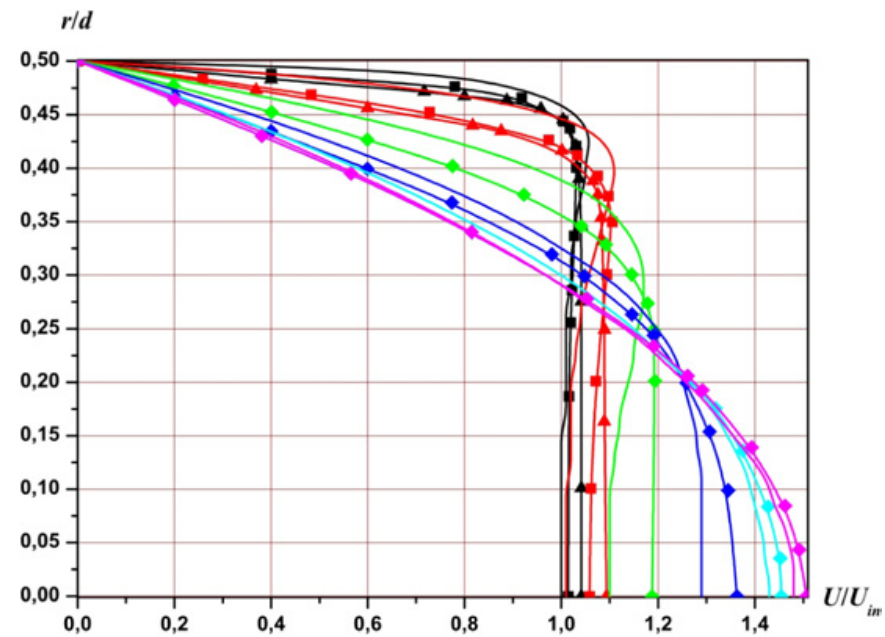

Figure 1. Velocity profiles in different sections of the flat channel. - • - - Theoretical and experimental results [3-5]; - Calculation by model [1].

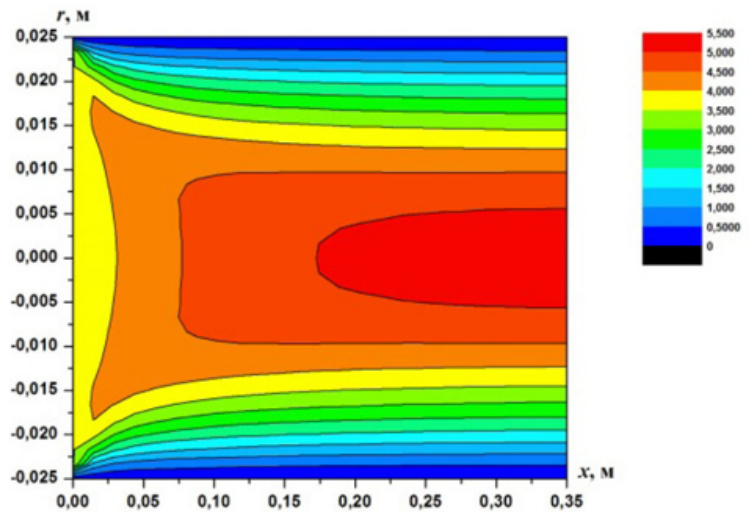

a)

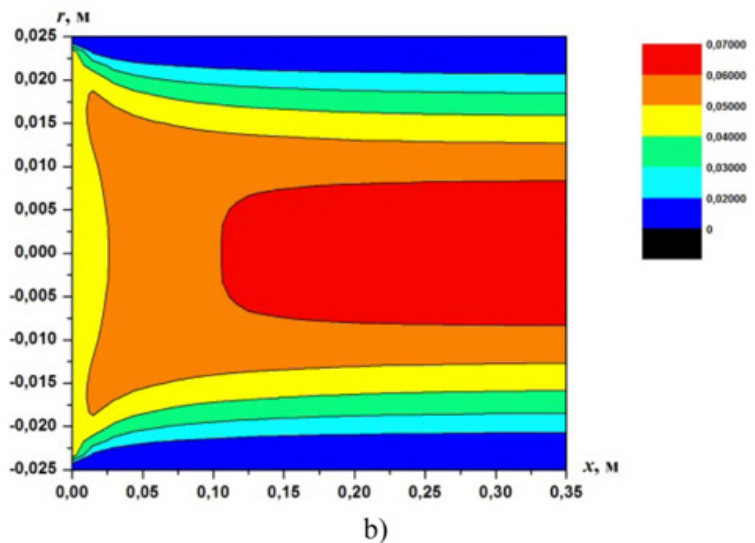

Figure 2. The longitudinal component of the velocity profiles of the glycerin flow (a) and air flow (b). 


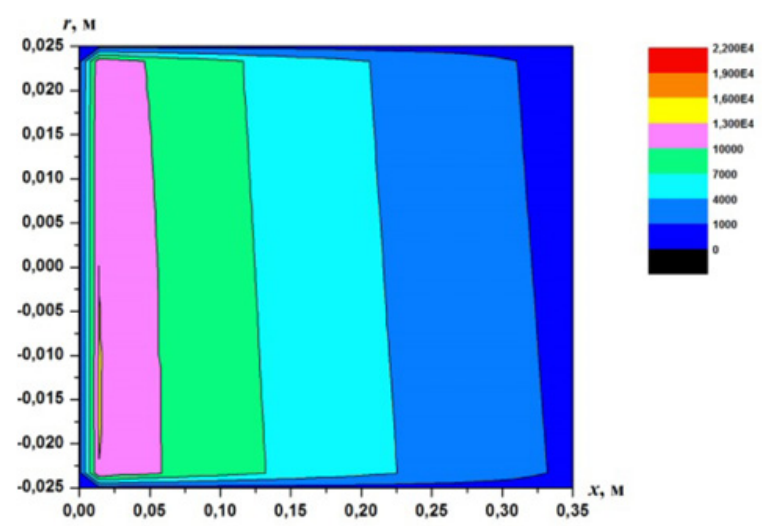

a)
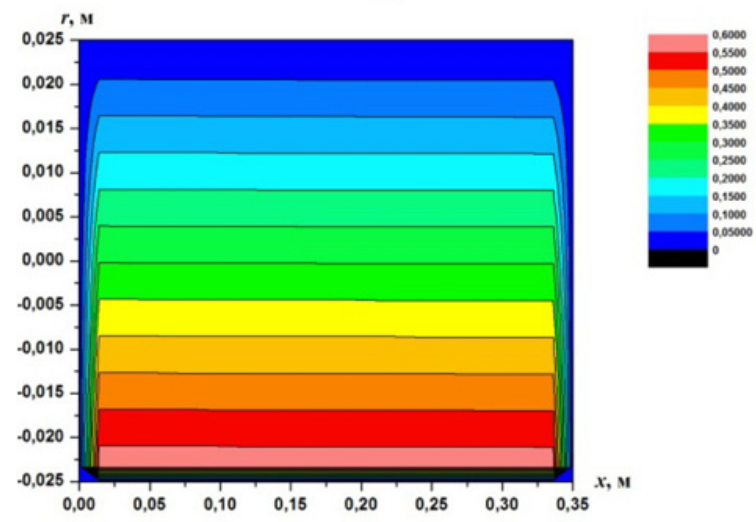

b)

Figure 3. Isobars of flow glycerin (a) and of flow air (b).

The profiles of the longitudinal velocity component in various sections along the channel are shown in Fig. 1 (curves correspond to values of $r / d=(0.044,0.208,0.833,3.333,7.50,13.5), r$ is the current value of the radius, $d$ is a diameter). Comparative analysis showed that velocity profiles are differ slightly for different environments in different channel sections in this range of output velocities $u_{\text {in }}=\left(4.53 \cdot 10^{-6} \div 3.55\right) \mathrm{m} / \mathrm{sec}$. Mismatch ofthe obtained resultsand given data in [3-5] in the range of sections $r / d=(0.208 \div 3.333)$ is connected to the use of border functions for the implementation of the numerical solution algorithm.

The velocity and pressure fields were analyzed on the basis of the proposed model and algorithm of numerical implementation. The structure features of flows in the channel of mediums with significant viscous interaction between the phases were identified. There are the results of one of calculation variants for the glycerin flow and air flow in the channel when diameter $d=0.05 \mathrm{~m}$ and a length $L=0.35 \mathrm{~m}$ in Figs. 2 and 3. It was found that the flow of air and water have a layered structure, in contrast to the glycerin flow in considered conditions. This is because the glycerin is more dense and viscous medium compared to air and water.

Thus, the test results showed that the proposed mathematical model and implementation algorithm are adequate to the physics of the investigated process. 


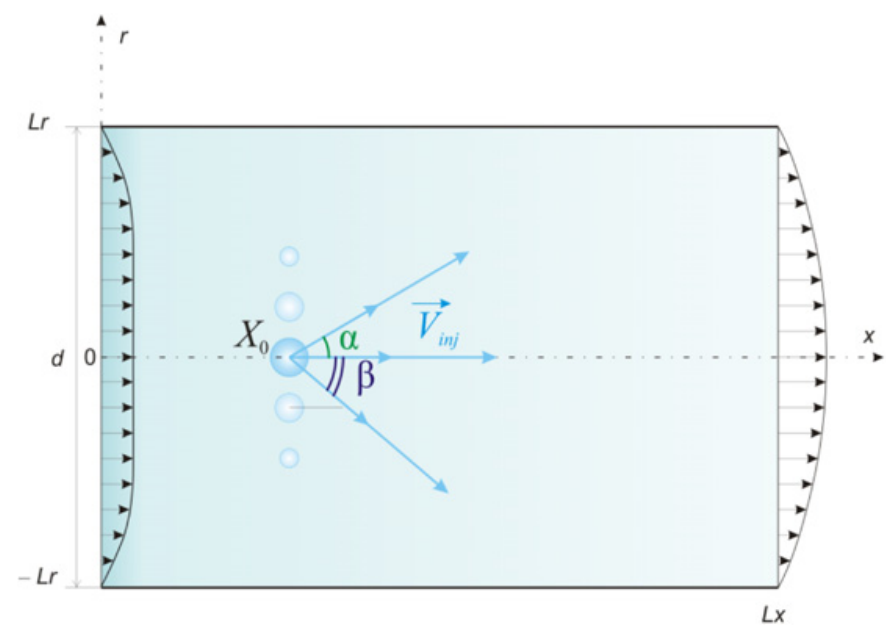

Figure 4. Scheme of solution area.

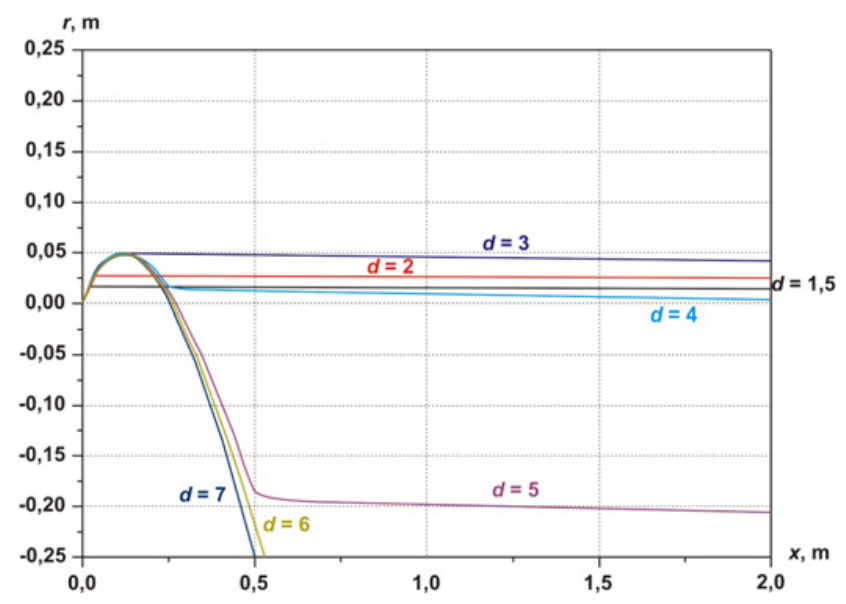

Figure 5. Trajectory of movement of various initial droplet diameter, $\mathbf{V}_{i n j}=\{1 ; 1\} \mathrm{m} / \mathrm{s}$.

\section{Analysis of the structure of gas-vapor mixture flow during the injection of water droplets in the channel}

The proposed algorithm of mathematical modeling was used for numerical analysis of the dynamics and evaporation of the droplet of water injected into a gas-vapor flow. Parametric study was conducted under the following conditions: channel length is $L_{x}=2 \mathrm{~m}$; channel cross-sectional radius is $L_{r}=0.25 \mathrm{~m}$; gas mixture temperature is $T_{f}=100^{\circ} \mathrm{C}$; initial flow velocity is $u_{\text {in }}=10 \mathrm{~m} / \mathrm{sec}$; the initial temperature of the injected droplet of water is $T_{i n j}=20^{\circ} \mathrm{C}$. Scheme of solution area is shown in Fig. 4.

The initial parameters of the droplet were varied under studies: diameter is $d_{p 0}=(1 \div 7) \cdot 10^{-3} \mathrm{~m}$, coordinates are $X_{0}=\{0 ;(-0.2 \div 0.2)\} \mathrm{m}$, the velocity of injection droplet is $\mathbf{V}_{i n j}\left(V_{x p}=1 \mathrm{~m} / \mathrm{s}, V_{r p}=\right.$ $(-0.5 \div 3) \mathrm{m} / \mathrm{s} ; V_{x p}$ and $V_{r p}$ are longitudinal and transverse components of the velocity vector).

Motion path droplet which injected into the gas-vapor flow under different initial conditions are shown in Figs. 5 and 6. 


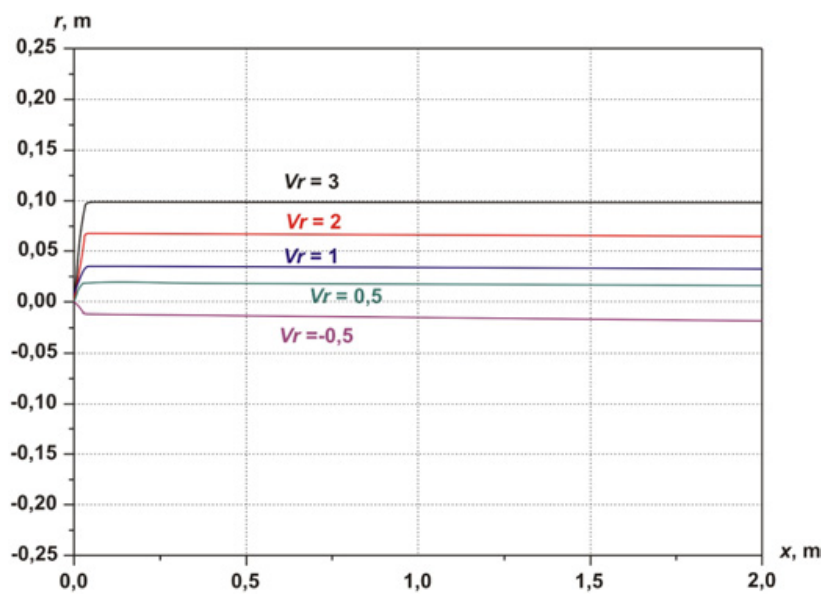

Figure 6. Droplets trajectories under the different initial velocity, $d_{p 0}=2 \cdot 10^{-3} \mathrm{~m}$.

\section{Conclusion}

Analysis of the results showed that the water droplets with initial diameters less than $5 \mathrm{~mm}$ was completely vaporized at injection into the gas-vapor mixture flow on the axis of symmetry of the channel (Fig. 5) for the given parameters of the study. It is necessary increasing the gas-vapor flow velocity for the complete evaporation of droplets of larger diameter in order to avoid their deposition on the channel walls with further formation of a liquid film.

Droplet with diameter $d_{p 0}=2 \cdot 10^{-3} \mathrm{~m}$ completely evaporate in the axial region when their injection from the origin into the channel have an initial velocity $V_{i n j}=\{1 ;(-0,5 \div 3)\} \mathrm{m} / \mathrm{s}$ (Fig. 6).

We obtained that droplets with initial diameter $\mathrm{d}_{p 0} \approx 5 \mathrm{~mm}$ can as evaporate so and can precipitate on the channel walls in this velocities range of injection. Droplets with initial diameter $d_{p 0} \geq 6 \mathrm{~mm}$ are precipitated completely on the channel wall.

Work is done at the expense of subsidies in the framework of the program to improve the competitiveness of TPU.

\section{References}

[1] E. Maslov, I. Zharova,V. Terekhov. Russian Physics Journal, 53, 182 (2010)

[2] H. Versteeg, W. Malalasekera. An introduction to computational fluid dynamics. The finite volume method (Longman Scientific \& Technical, 1995)

[3] I. Vinogradova, V. Zubkov. Mathematical Modeling, 14, 3 (2002)

[4] N. Buleev, G. Timukhin. Journal of Applied Mechanics and Technical Physics, 3,126 (1967)

[5] Y. Wang, P. Longwell. American Institute Chemical Engineering Journal, 10, 147 (1964) 